Received: 2017.11.20

Accepted: 2018.04.03

Published: 2018.08 .19

\title{
Overexpression of Neuregulin-1 (NRG-1) Gene Contributes to Surgical Repair of Brachial Plexus Injury After Contralateral C7 Nerve Root Transfer in Rats
}

Authors' Contribution: Study Design A Data Collection B Statistical Analysis C Data Interpretation D Manuscript Preparation E Literature Search F Funds Collection G

Corresponding Authors: Source of support:

\author{
BC 1 Zong-Qiang Wang \\ AB 2 Dian-Hui Xiu \\ CD 2 Gui-Feng Liu \\ $B C$ 3,4 Jin-Lan Jiang
}

\author{
1 Medical Department, China-Japan Union Hospital of Jilin University, Changchun, \\ Jilin, P.R. China \\ 2 Department of Radiology, China-Japan Union Hospital of Jilin University, \\ Changchun, Jilin, P.R. China \\ 3 Department of Orthopedics, China-Japan Union Hospital of Jilin University, \\ Changchun, Jilin, P.R. China \\ 4 Scientific Research Center, China-Japan Union Hospital of Jilin University, \\ Changchun, Jilin, P.R. China
}

Background: Surgeons usually transfer the contralateral C7 to the median nerve on the injured side via a nerve graft to recover sensation and movement in a paralyzed hand. The purpose of our study was to determine whether NRG-1 affects the recovery of nerve function in brachial plexus injury after contralateral $\mathrm{C} 7$ nerve root transfer in a rat model.

Material/Methods: An injury model of left brachial plexus and contralateral C7 nerve root transfer was established. Four weeks after the operation, NRG-1 expression was examined by reverse transcription quantitative polymerase chain reaction and Western blot analysis. The diameter rate differences of the healthy limb and affected limb were estimated. The postoperative mass of the left latissimus dorsi, triceps, extensor carpi radialis brevis, and musculus extensor digitorum were examined. The number of nerve fibers and typical area of the affected side were assessed. Postoperative left motor nerve conduction velocity (MNCV) and motor nerve action potential (MNAP) were tested by use of a biological information recording and collecting system.

Results: Eukaryotic expression plasmid of pcDNA4/myc/A-NRG-1 was successfully constructed, and NRG-1 was overexpressed. Compared with the model group, the NRG-1 group had a lower rate of differences of the limbs; higher mass of left latissimus dorsi, triceps, extensor carpi radialis brevis, and musculus extensor digitorum; more nerve fibers and larger typical area in the affected side, left MNCV, and MNAP; and wider CSA of the left triceps.

Conclusions: These results demonstrated that NRG-1 can promote recovery of nerve function in brachial plexus injury after contralateral C7 nerve root transfer in rats.

MeSH Keywords: $\quad$ Brachial Plexus • Muscular Atrophy, Spinal • Radiology

Full-text PDF: https://www.medscimonit.com/abstract/index/idArt/908144

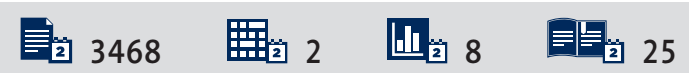




\section{Background}

Brachial plexus injuries have been increasing due to the growing numbers of motor vehicle accidents, extreme sporting accidents, and improper obstetric operations [1,2]. Brachial plexus injuries occurring in patients with displacement during an operation have been well documented and discussed in the medical literature [3]. As a destructive injury leading to partial or whole muscle denervation of the upper limb, brachial plexus injuries have been treated by many surgical methods, including neurolysis, nerve transfer, nerve grafting, and free-functioning gracilis muscle transfers [4]. However, these methods are not effective enough for reconstruction and restoration of the brachial plexus [5]. To overcome the shortcomings of methods, such as finite resources of obtainable donor nerves and inadequate myelinated axon fibers of the donor nerves, Gu et al. conducted contralateral $\mathrm{C} 7$ nerve root transfer to restore functional hand grip [6].

During a contralateral C7 nerve root transfer operation, the injured nerve on the injured side is repaired by transferring the partial or total seventh cervical nerve on the uninjured side using a nerve graft with more myelinated nerve fibers than in other available donor nerves, providing sufficient power for neurotization [7]. According to Yang et al., functional free-muscle repair and nerve transfer have been commonly treated with contralateral C7 nerve root transfer, especially in some Asian countries [8]. Due to its better recovery function, contralateral C7 nerve root transfer has become one of the most commonly conducted operations in treating brachial plexus avulsion injuries [9]. As a multipurpose and versatile protein, Neuregulin-1 (NRG-1) has great isoform signaling in an autocrine, paracrine, or juxtacrine manner, playing a basic role in developing the peripheral nervous system and in nerve repair, indicating that NRG-1 can promote functional repair after postoperative injuries [10]. According to Curr et al., NRG1 is required for effective nerve repair and remyelination [11]. However, there is still much variation in the procedures used in treatment of brachial plexus injuries using contralateral $\mathrm{C} 7$ nerve root transfer, such as differences in age, time of reconstruction, length of grafts, and the specific target muscle group [12]. Based on the above research, the present study estimated the effects of NRG-1 on the recovery of nerve function in brachial plexus injury after contralateral $\mathrm{C} 7$ nerve root transfer in a rat model.

\section{Material and Methods}

\section{Ethics statement}

This study was performed in accordance to the ethics of scientific research conducted using experimental animals under the approval of our Clinical Management Committee.

\section{Study subjects}

A total of 48 male Sprague-Dawley (SD) rats weighing 160$180 \mathrm{~g}$, provided by our institution's Animal Center, were selected and kept in a clean environment with humidity of $49.7 \%$ and temperature at about $22^{\circ} \mathrm{C}(12 \mathrm{~h}$ light and $12 \mathrm{~h}$ dark) for 1 week of adaptive feeding before experiments.

\section{Preparation and identification of pcDNA4/myc/A-NRG-1 plasmid}

Eukaryotic expression vector of $\mathrm{pcDNA} 4 / \mathrm{myc} / \mathrm{A}$ was purchased in Genentech. Polymerase chain reaction (PCR) was implemented to amplify hNRG-1 fragment (about 900 bp) from cDNA. A gel extraction kit (Shanghai Sangon Biological Engineering Technology \& Services Co., Ltd., Shanghai, China) was used to purify hNRG-1 segment. T4 DNA ligase was used to connect the double-enzyme vector, which was detached by endonuclease HindIII and Xhol and hNRG-1 fragment (the endonucleases and T4 DNA ligase were purchased from NEB Company, Beijing, China). Products obtained after connection were transformed into $\mathrm{DH} 5$ alpha-competent cells through thermal stimulation method (Kangwei Century Technology, Co., Ltd., Beijing, China). After the collection of monoclones, positive clones were identified by RT-qPCR and then amplified and cultured. Plasmid was extracted by a plasmid extraction kit and sent for sequencing and pair analysis (Shanghai Biosune Biotechnology Co. Ltd., Shanghai, China). Clones with right sequences indicated successful construction of objective plasmids. An ultraviolet spectrophotometer (Thermo Fisher Scientific, Massachusetts, USA) was used to assess concentration of cloned plasmids with right sequences. The PRC primer for plasmid preparation and identification: hNRG-1: upstream: 5'-GAGGATCCCCGGGTAC CGGTCGCCACCATG GAGATTATTCCCCAG-3'; downstream: 5'-TCACCATGGTGGCGACCG GTTCAGGCAG AGACAGAAAG-3'. The bold base sequences were enzyme sequences.

\section{Model establishment and grouping}

The injury model of left brachial plexus and contralateral $C 7$ nerve root transfer was built using 32 SD rats: After abdominal anesthesia of $1 \%$ Carbitol (5 mg/kg, Shanghai Sangon Biological Engineering Technology \& Services Co., Ltd., Shanghai, China), a $15-\mathrm{mm}$ incision was made along the left clavicle beginning from the left sternoclavicular joint to expose the left brachial plexus after severing the collarbone. Then, the roots from C 5 to T1 were severed from the spine. Brachial plexus injuries were made by cutting down about $2 \mathrm{~mm}$ of the nerve from $\mathrm{C} 5$ to the end of $\mathrm{T} 1$. The right brachial plexus was exposed in the same way and the $\mathrm{C} 7$ nerve was severed at the separating point. The rest of the right $\mathrm{C} 7$ nerve and the left $\mathrm{C} 7$ nerve were bridged with a $10-\mathrm{mm}$ nerve (from the left ulnar nerve) (Figure 1). An Alzet 


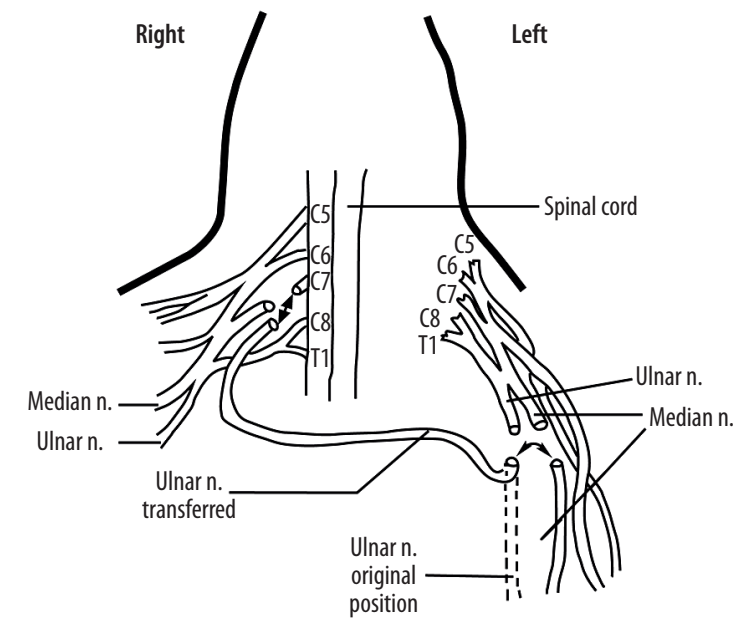

Figure 1. Sketch map of avulsion of left brachial plexus and contralateral C7 nerve root transfer operation.

micro-osmotic pump (Product Type 2004 Cupertino, USA) was placed alongside the spine with the model and NRG-1 groups of 16 pumps for each. After the wound was stitched, each postoperative rat was housed in a single cage with drinking water provided. Their bladders were pressed for urination every morning and every night until the rats recovered automatic urination. Another 16 rats were assigned to the sham group following the same operation as above but without avulsion of the left brachial plexus and contralateral C7 nerve root transfer.

Alzet micro-osmotic pump was constructed according to the instructions and then added with a mixture of recombinant plasmids and $15 \mathrm{uL}$ of Lipofectamine TM 2000 (Invitrogen, California, USA) with a ratio of 1: 3. Then, phosphate-buffered saline (PBS) was added to the mixture to $200 \mu \mathrm{L}$. This pump was assigned to the NRG-1 group. In the model group, $5 \mu \mathrm{g}$ of blank plasmid was added to an Alzet micro-osmotic pump with the same other conditions as the NRG-1 group. The pumps were soaked in PBS in order to improve transfection efficiency.

\section{Reverse transcription polymerase chain reaction (RT-qPCR) and Western blot analysis}

Four weeks after the operation, 6 rats of each group were sacrificed. The left brachial plexus was exposed as discussed above. After being peeled from the spine to nerve endings, the nerve was immediately put into a homogenizer for milling and then divided into 2 parts.

One was promptly used to extract the total RNA from nerve tissues without RNA enzymes according to the instructions of the Trizol kit (Invitrogen, California, USA). We used a reverse transcription kit (Takara Biotechnology Ltd., Dalian,
China) to process the extracted total RNA to reverse the firststrand complementary DNA. The reverse transcription system $(10 \mu \mathrm{L})$ was as follows: $5.5 \mu \mathrm{L}$ total RNA, $2 \mu \mathrm{L} 5 \times$ buffer, $1 \mu \mathrm{L}$ dNTP, $0.5 \mu \mathrm{L}$ Oligo (dT), $0.5 \mu \mathrm{L}$ RNase inhibitor, $0.5 \mu \mathrm{L}$ M-MLV reverse transcriptase, and $1 \mu \mathrm{L}$ RNase-free water. The mixture was water-bathed at $42^{\circ} \mathrm{C}$ for $60 \mathrm{~min}$ and then heated at $95^{\circ} \mathrm{C}$ for $2 \mathrm{~min}$ to stop the reaction. The first-chain CDNA was separated and stored at $-20^{\circ} \mathrm{C}$. Fragments were amplified with the following primers (Ex Taq DNA polymerase was purchased from Takara Biotechnology Ltd., Dalian, China): hNRG-1: upstream: 5'-GAGGATCCCCGGGTACCGGTCGCCACCA TGGAGATTATTCCCCAG-3'; downstream: 5'-TCACCATGGTGGCGAC CGGTTCAGGCAGAGACAGAAAG-3'. $\beta$-actin: upstream: 5'-TGTGCTATGTTGCCCTAGACTTC; downstream: CGGACTCATC GTACTCCTGCT-3'. PCR reactions were performed as follows: pre-denaturation at $94^{\circ} \mathrm{C}$ for $5 \mathrm{~min}, 35$ cycles of denaturation at $94^{\circ} \mathrm{C}$ for $30 \mathrm{~s}$, annealing at $58^{\circ} \mathrm{C}$ for $40 \mathrm{~s}$, extension at $72^{\circ} \mathrm{C}$ for $30 \mathrm{~s}$, and extension again at $72^{\circ} \mathrm{C}$ for $5 \mathrm{~min}$. The PCR products were assessed using electrophoresis on $2 \%$ agarose gels. GAPDH served as the internal reference gene.

The other part was used to extract protein (Roche), followed by protein concentration determination by use of an ultraviolet spectrophotometer (Thermo Fisher Scientific, Massachusetts, USA). Protein was transferred to a polyvinylidene difluoride (PVDF) membrane at constant current after $10 \%$ sodium dodecyl sulfate (SDS) gel electrophoresis. Then, the membrane was blocked with Tris-buffered saline with tween (TBST) containing 5\% skim milk at room temperature for $1 \mathrm{~h}$ and then incubated overnight at $4^{\circ} \mathrm{C}$ with anti-NRG-1 (1: 200, sc-393006, molecular weight of 26-71 kd, Santa Cruz Biotechnology, Inc., Shanghai, China). After rinsing 3 times with TBST (10 $\mathrm{min}$ for each time), the membrane was incubated at room temperature for $1 \mathrm{~h}$ with horseradish peroxidase (HRP)-conjugated secondary antibody (1: 5000) (MultiSciences [Lianke] Biotech Co., Ltd., Hangzhou, Zhejiang, China), followed by development via chemiluminescent substrates.

\section{Diameter measurement of forelimbs, muscle weighing, and HE staining for triceps}

Diameter measurement of forelimbs was as follows: at 1 month after the operation, rats were anesthetized by abdominal injection of $1 \%$ pentobarbital sodium $(5 \mathrm{mg} / \mathrm{kg}$, Sangon Biotech, Shanghai, China). Forelimb diameters were measured before extraction of muscle and brachial plexus. The maximum transverse diameters of the inner and outer sides of the knee (the maximum transverse diameter of the medial and lateral elbow) and the diameters of forelimbs were measured using calipers. Because nerve injuries in the affected limbs may lead to muscular atrophy, the diameter rate differences of the healthy limbs and the affected limbs were used to estimate the degree of muscular atrophy. The rate differences were calculated 


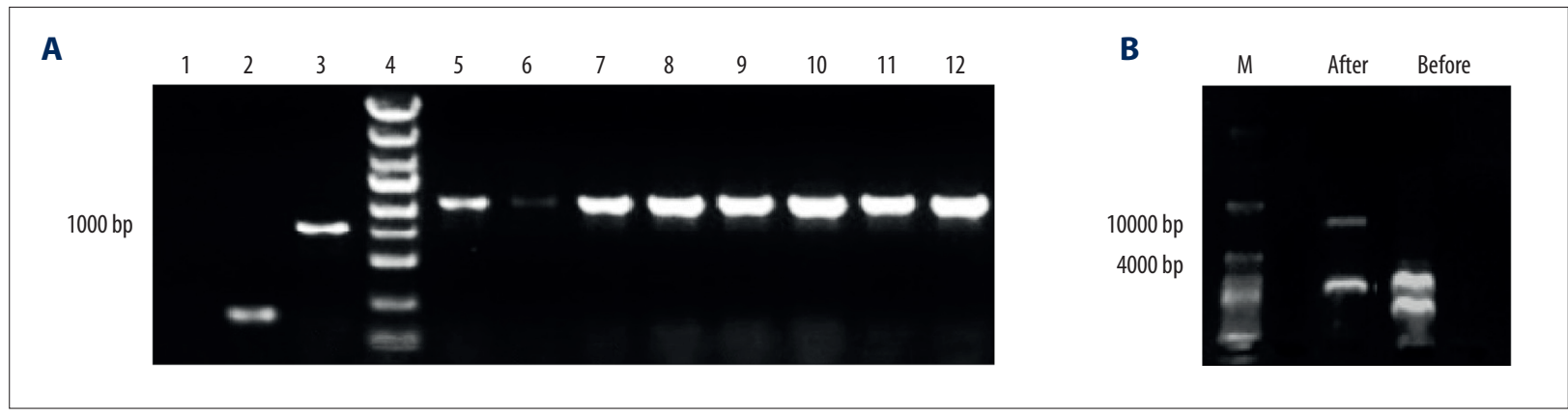

Figure 2. NRG-1 recombinant plasmid was constructed by double-enzyme digestion and identified by PCR. (A) 8 clones (5-12) were selected randomly and identified by PCR, 1: blank control, 2: empty plasmid control, 3: positive control (GAPDH), 4: marker; (B) plasmid enzyme detachment, M: marker, after: after HindIII and Xhol double-enzyme digestion, before: before doubleenzyme digestion.

with the following formula: rate difference $=[$ (diameter of the healthy limb - diameter of the affected limb) $\div$ diameter of the healthy limb]×100.

One month after the operation, rats were anesthetized in the same way. A 15-mm incision was made along the left clavicle beginning from the left sternoclavicular joint to expose left brachial plexus and left limb muscle. Left latissimus dorsi, extensor carpi radialis brevis, musculus extensor digitorum, and triceps were carefully extracted and blotted by absorbent paper so as not to affect the muscle mass. The muscle weight was immediately determined by analytical balance and then recorded. $\mathrm{HE}$ staining for triceps was as follows: triceps were cross-cut, fixed with $4 \%$ poly formaldehyde, then rinsed with water, dehydrated with ethanol of gradient concentration, and finally embedded in paraffin. The triceps were sectioned at $5-\mu \mathrm{m}$ thick, stained by $\mathrm{HE}$ staining, and then mounted for observation under a microscope.

\section{Examination of motor nerve conduction velocity (MNCV) and motor nerve action potential (MNAP)}

The left brachial plexus was removed and placed in Ringer solution at $37.8^{\circ} \mathrm{C}$ for $10 \mathrm{~min}$. MNCV and MNAP were recorded by the Medlab-u/4 cs (V6.0) biological signal collecting and processing system (Nanjing Beauty Star Biotechnology Co., Ltd., Nanjing, Jiangsu, China). The stable brachial plexus nerve samples were placed on the electrode of the nerve-shielding box with the central end connected to the stimulating electrode and the peripheral end connected to the guide electrode. Electrical pulses were generated by stimulating electrodes: $5 \mathrm{~V}, 3.1 \mathrm{~ms}$, $1 \mathrm{~s}$ of major cycle, 1 time of pulse after $10 \mathrm{~ms}$ of interval, followed with $20 \mathrm{~ms}$ of time delay. The results were recorded and stored for estimation of nerve function.

\section{Toluidine blue staining}

Four weeks after the operation, the left brachial plexus tissues of the rats were collected for fiber counting. Tissues
$5 \mathrm{~mm}$ from the proximal and distal junctions were taken as samples. The samples were fixed with glutaraldehyde (2.5\%), prepared by arsenate buffer solution, then washed with sodium arsenate buffer solution ( $0.2 \mathrm{~mol} / \mathrm{L}, \mathrm{pH} 7.4)$, fixed with $1 \%$ osmium acid. The samples were dehydrated and embedded in epoxy resin. The nerve samples were cut into $0.5-\mu \mathrm{m}$ thick sections and stained with toluidine blue. The detection and observation of cross-sectional area were performed using Leica DWLB2 (×400, Germany) and QWin Histomorphometry System (Leica, Germany) was used for nerve fiber counting.

\section{Statistical analysis}

SPSS 18.0 software (SPSS Inc., Chicago, IL, USA) was used for statistical analysis. Measurement data are displayed as mean \pm standard deviation. The comparison of the measurement data with the normal distribution between the 2 groups was performed using the unpaired double-tailed $t$ test. $p<0.05$ was considered as statistically significant.

\section{Results}

\section{Recombinant plasmid of NRG-1 overexpression is successfully constructed}

After connecting NRG-1 fragment and pcDNA4/myc/A, 8 plasmids were selected to clone to produce correctly connected plasmids. RT-qPCR was used to identify the cloned plasmids. As shown in Figure 2A, except for number 6, other plasmids amplified about 1300 bands. Number 12 was chosen for expanded cultivation, plasmid extraction, and further identification by double-enzyme digestion. Gel electrophoresis with $1.5 \%$ agarose gel was conducted on products produced before and after enzyme digestion. Plasmids, recombined by endonuclease HindIII and Xhol, showed one Nrg-1 band by double-enzyme digestion (1300 bp) and one vector band (about $5000 \mathrm{bp}$ ). Results of double-enzyme digestion indicated that 


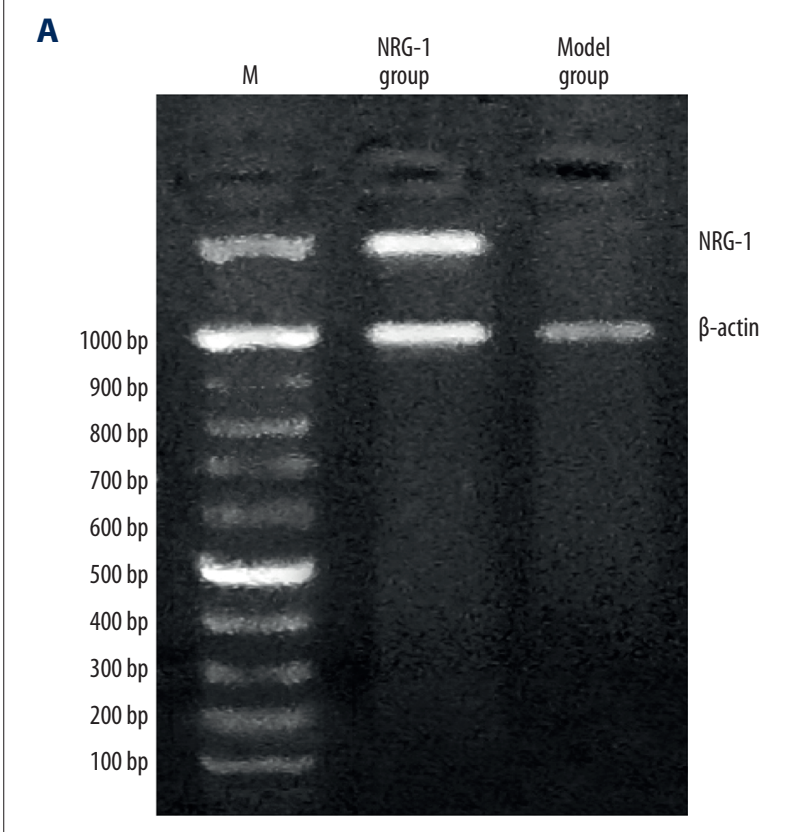

B

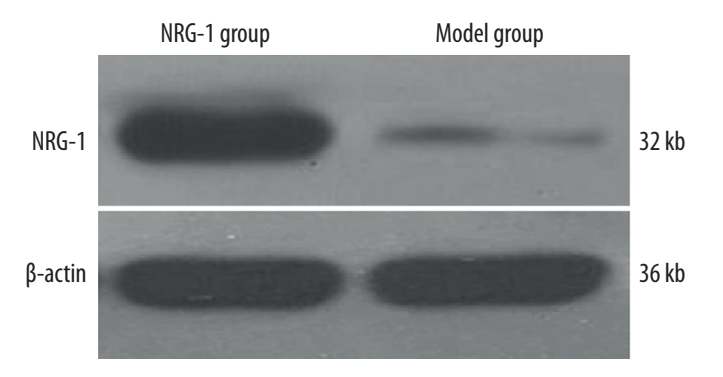

Figure 3. mRNA and protein expressions of NRG-1 were up-regulated in brachial plexus of rats in the NRG-1 group. (A) RT-PCR detection results of NRG-1 mRNA in brachial plexus of 2 groups; (B) Western blot analysis results of NRG-1 protein expression in brachial plexus of 2 groups.

NRG-1 was successfully connected (Figure 2B). Sequencing outcomes further demonstrated the successful construction of NRG-1 recombined plasmid.

\section{NRG-1 was successfully overexpressed in brachial plexus in the NRG-1 group}

To identify the successful overexpression of NRG-1 in brachial plexus in the NRG-1 group, the affected side of the brachial plexus was severed from the rats in the model and NRG-1 groups at 4 weeks after operation. Then, RNA and protein were extracted. Effects of NRG-1 mRNA and protein expressions of brachial plexus in the 2 groups were detected by RTqPCR, DNA gel electrophoresis, and Western blot analysis. NRG-1 mRNA was only expressed in brachial plexus in experimental rats (Figure $3 \mathrm{~A}$ ). To further investigate the expression of NRG-1 in the 2 groups, Western blot analysis was used to examine the NRG-1 protein expression. NRG-1 was obviously expressed in the NRG-1 group, while little expression was found in the model group (Figure $3 \mathrm{~B}$ ). These results indicate that NRG-1 is successfully overexpressed in brachial plexus in the NRG-1 group.

\section{NRG-1 overexpression can relieve muscular atrophy in the affected side}

The rate differences in the model and NRG-1 groups were larger than in the sham group (Figure 4), indicating the successful

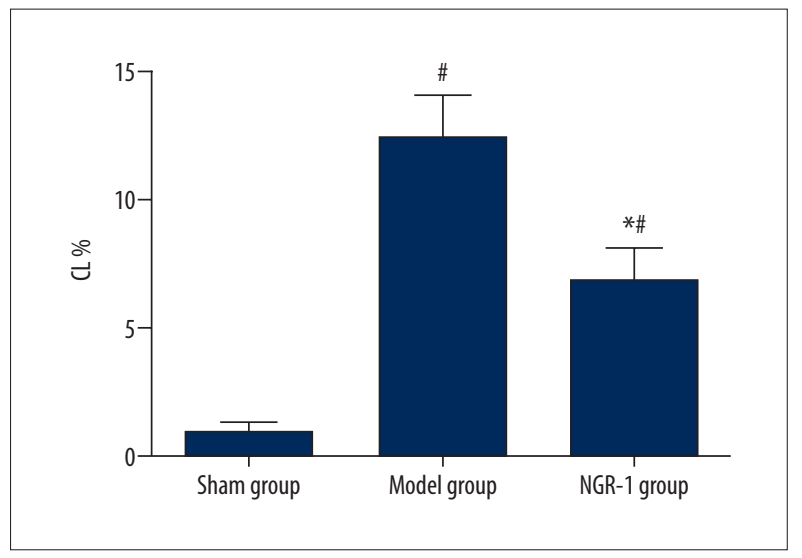

Figure 4. Rate differences between the diameter of the healthy limb and the affected limb of the rats in the NRG-1 group was lower than that in the model group. Rate difference $=[$ (diameter of the healthy limb - diameter of the affected limb) $\div$ diameter of the healthy limb] $\times 100$; ${ }^{\#} p<0.05$ vs. the sham group; ${ }^{*} p<0.05$ vs. the model group. The data are presented as mean \pm standard deviation, analyzed by $t$ test. The experiment was independently repeated 3 times.

construction of the injury model of brachial plexus. The rate differences of the NRG-1 group were significantly lower than in the model group (both $p<0.05$ ). The results illustrate that NRG-1 overexpression can relief muscular atrophy in the affected side. 
Table 1. Comparisons of muscle mass in the affected side in the model group, the NRG-1 group and the sham group ( $n=10$, $g$ ).

\begin{tabular}{|cccc}
\hline Kinds of muscle & Sham group & Model group & NRG-1 group \\
\hline Latissimus dorsi & $0.354 \pm 0.026$ & $0.137 \pm 0.020^{\#}$ & $0.255 \pm 0.021^{\text {*\# }}$ \\
\hline Ttriceps & $0.371 \pm 0.021$ & $0.250 \pm 0.031^{\#}$ & $0.335 \pm 0.023^{* \#}$ \\
\hline Extensor carpi radialis brevis & $0.311 \pm 0.034$ & $0.139 \pm 0.014^{\#}$ & $0.251 \pm 0.022^{* \#}$ \\
\hline Musculus extensor digitorum & $0.369 \pm 0.030$ & $0.210 \pm 0.016^{\#}$ & $0.301 \pm 0.027^{\text {*\# }}$ \\
\hline
\end{tabular}

NRG-1 - Neuregulin-1; " represents the comparison with the sham group; * represents the comparison with the model group, all $p<0.05$.
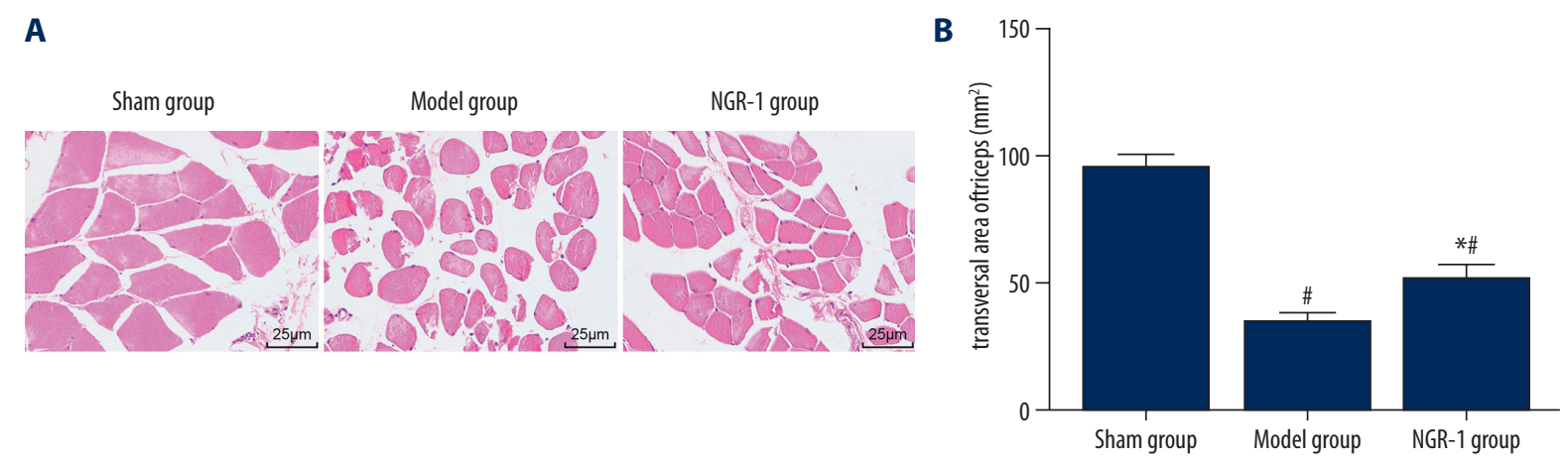

Figure 5. Cross-sectional area of triceps in the affected side was enlarged after HE staining in the NRG-1 group (400x). (A) HE staining images of the cross-sectional area of triceps in the affected sides of rats in the sham, model, and NRG-1 groups; (B) HE staining histogram of the cross-sectional area of triceps in the affected sides of rats in the sham, model, and NRG-1 groups. ${ }^{\#} p<0.05$ vs. the sham group; ${ }^{*} p<0.05$ vs. the model group. The data are presented as mean \pm standard deviation, analyzed by $t$ test. The experiment was independently repeated 3 times.

\section{Overexpressed NRG-1 can increase the mass of left latissimus dorsi, triceps, extensor carpi radialis brevis, and muscle extensor digitorum after contralateral $\mathrm{C7}$ nerve root transfer}

Muscle mass associated with brachial plexus was another indicator of muscle atrophy. In order to further evaluate the degree of muscle atrophy, left latissimus dorsi, triceps, extensor carpi radialis brevis, and musculus extensor digitorum were extracted and weighed 4 weeks after the operation. As shown in Table 1, the mass of left latissimus dorsi, triceps, extensor carpi radialis brevis, and musculus extensor digitorum in the model and NRG-1 groups were significantly greater than in the sham group (all $p<0.05$ ).

\section{Up-regulated NRG-1 can promote the repair of brachial plexus function after contralateral $\mathrm{C7}$ nerve root transfer}

Muscle atrophy may lead to a reduction in muscle fiber CSA. Using triceps in the affected side as an example, we used $\mathrm{HE}$ staining to evaluate the triceps CSA. The triceps CSA in the affected side in the NRG-1 group was obviously larger than that in the model group (Figure 5$)(p<0.05)$, indicating that overexpression of NRG-1 can promote the repair of brachial plexus function after contralateral C7 nerve root transfer.

\section{Overexpression of NRG-1 can increase MNCV in the affected side after contralateral $\mathrm{C} 7$ nerve root transfer}

Further evaluation of NRG-1 effects on the repair of brachial plexus function after contralateral C7 nerve root transfer was conducted with electrophysiological parameters. The MNCV of the sham group, without brachial plexus injury, was faster than in the other 2 groups, and the MNCV of the NRG-1 group was significantly higher than in the model group (all $p<0.05$ ) (Figure 6).

\section{Elevated expression of NRG-1 can increase the MNAP of the affected side after contralateral $C 7$ nerve root transfer}

The other electrophysiological index for evaluating nerve function is MNAP. Under a certain stimulus, the greater MNAP reflected the better nerve functions. MNAP of the sham group without brachial plexus injury was faster than in the other 2 groups, and MNAP of the NRG-1 group was significantly higher than in the model group (all $p<0.05$ ) (Figure 7). These results, together with the results of MNCV, indicated that NRG-1 can 


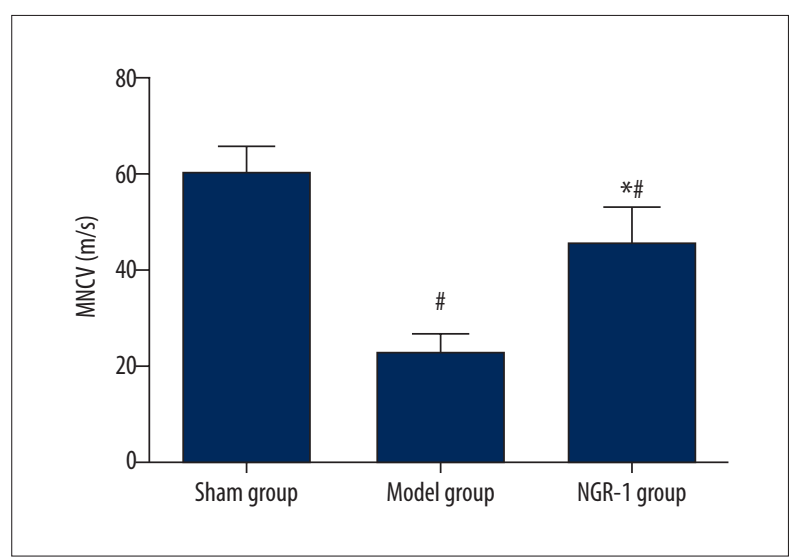

Figure 6. MNCV in the affected side of rats in the NRG-1 group was enhanced compared to that in the model group. \# $p<0.05$ vs the sham group; ${ }^{*} p<0.05$ vs the model group. The data are presented as mean \pm standard deviation, analyzed by $t$ test. The experiment was independently repeated 3 times.

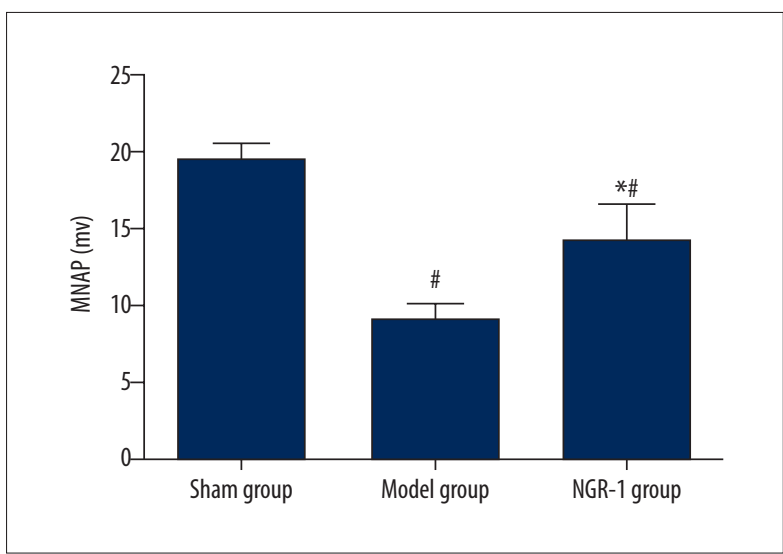

Figure 7. MNAP in the affected side of rats in the NRG-1 group was elevated compared to that in the model group. ${ }^{\#} p<0.05$ vs. the sham group; ${ }^{*} p<0.05$ vs. the model group. The data are presented as mean \pm standard deviation, analyzed by $t$ test. The experiment was independently repeated 3 times.

Table 2. Comparisons of the number of nerve fibers and cross-sectional area in the sham, NRG-1 and model groups.

\begin{tabular}{cccc} 
& Sham group & Model group & NRG-1 group \\
\hline Numbers & $2545 \pm 108$ & $1677 \pm 95^{\#}$ & $2217 \pm 100^{* \#}$ \\
\hline Cross-sectional area $\left(\mu \mathrm{m}^{2}\right)$ & $125.72 \pm 11.14$ & $63.53 \pm 6.51^{\#}$ & $93.85 \pm 9.53^{* \#}$ \\
\hline
\end{tabular}

NRG-1 - Neuregulin-1; " represents the comparison with the sham group; * represents the comparison with the model group, all $p<0.05$.

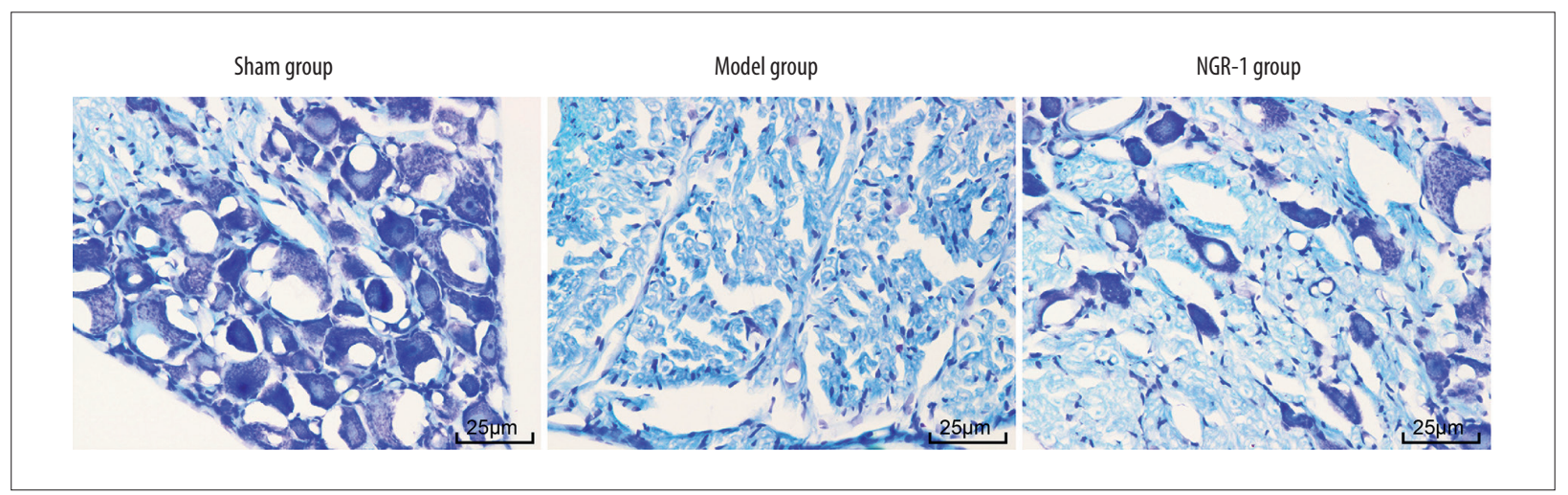

Figure 8. Nerve fibers and typical areas in the NRG-1 group were increased compared to that in the model group as determined by toluidine blue staining (400x).

promote the recovery of brachial plexus function after contralateral C7 nerve root transfer.

\section{Overexpressed NRG-1 can promote the recovery of brachial plexus function after contralateral $\mathrm{C} 7$ nerve root transfer}

The nerve fibers number and typical area of NRG-1 group were significantly larger than those of the model group $(p<0.05)$
(Table 2 and Figure 8). The aforementioned results suggest that overexpression of NRG-1 can promote the recovery of brachial plexus function after contralateral C7 nerve root transfer.

\section{Discussion}

As a very common disease, brachial plexus injury seriously influences life quality of humans [13]. This study estimated the 
effects of NRG-1 on the recovery of nerve function in brachial plexus injury after contralateral C7 nerve root transfer in a rat model.

Firstly, this study showed that the rate differences of the NRG-1 group were significantly lower than that of the model group, which illustrated that NRG-1 overexpression could relieve muscular atrophy in the affected side. The craniocaudal progression of birth brachial plexus injury may result in muscle atrophy, such as atrophy of the infraspinatus and the subscapularis [14]. As a growth factor delivered after nerve injury, NRG-1 sensitizes cells like erbB 2, 3, and 4 receptors on microglia, and promotes their survival, multiplication, and chemotaxis [15]. Belonging to a category of multipotent neuroprotective and anti-inflammatory growth factors, including acetylcholine receptor-inducing activities, heregulins, glial growth factors, and neu differentiation factors, NRG-1 was proved to function as a neuroprotectant in poisoning and other acute brain injuries [16]. An alpha-NRG-1-mediated autocrine or paracrine trophic loop, which was activated after denervation and immediately deactivated after nerve reconstruction, exists in skeletal muscles, suggesting a promising therapeutic target for preventing muscle atrophy [17].

The mass of left latissimus dorsi, triceps, extensor carpi radialis brevis, and musculus extensor digitorum in the model and NRG-1 groups were significantly greater than in the sham group, indicating that the degree of muscular atrophy in the NRG-1 group was lower than in the model group. Loss of muscle mass contributes to various diseases, including muscle wasting; therefore, developing a deeper understanding of the molecular mechanisms that control muscular atrophy is of great importance in developing new therapeutic methods [18]. According to the role of muscle imbalance in the pathogenesis of shoulder contracture after neonatal brachial plexus palsy, Soldado et al. found that the loss of subscapularis thickness was a cause of internal rotation muscle atrophy [19]. NRG1ErbB4 is relevant to spinal muscular atrophy because it plays a role in regulation of neural inflammation mediated by microglial in neurogenic diseases [20]. Our study also showed that the CSA of triceps in the affected side in the NRG-1 group was obviously larger than in the model group, showing that overexpression of NRG-1 can promote the recovery of brachial plexus function after contralateral C7 nerve root transfer. In accordance with the validity of estimating triceps brachii volume from single-MRI CSA before and after resistance training, Popadic et al. showed that using CSA for prediction of triceps brachii volume was promising, with the best area of CSA of $60 \%$ [21]. According to a study conducted by Narici et al., a reduction of CSA, representing a decrease of muscle size, was one of the space-correlated disused antigravity muscles that caused muscle atrophy [22].

MNCV and MNAP of the sham group were the highest in all 3 groups. MNCV and MNAP of the NRG-1 group were significantly higher than in the model group, demonstrating that NRG-1 can promote the recovery of brachial plexus function after contralateral C7 nerve root transfer. According to a study conducted by Coppey et al., the slowing of MNCV and the decrease in $\mathrm{Na}+/ \mathrm{K}+\mathrm{ATPase}$ activity in the sciatic nerve are signs of nerve disorders [23]. Higher MNCV in the NRG-1 group compared with the model group indicated the NRG-1 group had better brachial plexus function than the model group. Dawson and Scott demonstrated that when the nerve was irritated by a transient shock, an action potential (AP) could be acquired from the nerve at a higher level, providing significant practical value in nerve lesions [24]. In a recent study, Pondaag et al. investigated that amplitudes of nerve action potential and compound motor action potential to determine the severity of the nerve lesion [25]. As a multifunctional and versatile protein, NRG-1 plays a basic role in nerve repair and development of the peripheral nervous system, demonstrating that NRG-1 is a promising therapeutic target for therapeutic nerve regeneration [10].

\section{Conclusions}

Our study demonstrates that NRG-1 promotes the recovery of nerve function in brachial plexus injury after contralateral C7 nerve root transfer in a rat model, but does not define the mechanism that promotes the recovery of brachial plexus function. Due to limitations of effective time of the operation, the postoperative effects at 1 months or longer on the recovery of neurological function were not observed, and we also did not assess the effects on sensory nerve function. Further investigation is required due to the limitations of the current study, which still provides therapeutic guidance for brachial plexus function recovery of the affected side.

\section{Conflicts of interest.}

None. 


\section{References:}

1. Giuffre JL, Kakar S, Bishop AT et al: Current concepts of the treatment of adult brachial plexus injuries. J Hand Surg Am, 2010; 35: 678-88; quiz 688

2. Anguelova GV, Malessy MJ, van Zwet EW, van Dijk JG: Extensive motor axonal misrouting after conservative treatment of obstetric brachial plexus lesions. Dev Med Child Neurol, 2014; 56: 984-89

3. Uribe JS, Kolla J, Omar $\mathrm{H}$ et al: Brachial plexus injury following spinal surgery. J Neurosurg Spine, 2010; 13: 552-58

4. Wang L, Jiang Y, Lao J, Zhao X: Contralateral C7 transfer to lower trunk via the prespinal route in the repair of brachial plexus injury: An experimental study in rats. J Plast Reconstr Aesthet Surg, 2014; 67: 1282-87

5. Sammer DM, Kircher MF, Bishop AT et al: Hemi-contralateral C7 transfer in traumatic brachial plexus injuries: Outcomes and complications. J Bone Joint Surg Am, 2012; 94: 131-37

6. Tu YK, Tsai YJ, Chang CH et al: Surgical treatment for total root avulsion type brachial plexus injuries by neurotization: A prospective comparison study between total and hemicontralateral C7 nerve root transfer. Microsurgery, 2014; 34: 91-101

7. Yang G, Chang KW, Chung KC: A systematic review of contralateral C7 transfer for the treatment of traumatic brachial plexus injury: Part 1. Overall outcomes. Plast Reconstr Surg, 2015; 136: 794-809

8. Yang G, Chang KW, Chung KC: A systematic review of outcomes of contralateral C7 transfer for the treatment of traumatic brachial plexus injury: Part 2. Donor-site morbidity. Plast Reconstr Surg, 2015; 136: 480e-89e

9. Gao KM, Lao J, Zhao X, Gu YD: Outcome of contralateral C7 nerve transferring to median nerve. Chin Med J (Engl), 2013; 126: 3865-68

10. Gambarotta G, Fregnan F, Gnavi S, Perroteau I: Neuregulin 1 role in Schwann cell regulation and potential applications to promote peripheral nerve regeneration. Int Rev Neurobiol, 2013; 108: 223-56

11. Birchmeier C, Bennett DL: Neuregulin/ErbB signaling in developmental myelin formation and nerve repair. Curr Top Dev Biol, 2016; 116: 45-64

12. Stephenson JB $4^{\text {th }}$, Li R, Yan JG et al: Transhemispheric cortical plasticity following contralateral $C 7$ nerve transfer: A rat functional magnetic resonance imaging survival study. J Hand Surg Am, 2013; 38: 478-87

13. Mei RJ, Xu YY, Li Q: Experimental study on mechanical vibration massage for treatment of brachial plexus injury in rats. J Tradit Chin Med, 2010; 30: 190-95
14. Van Gelein Vitringa VM, Jaspers R, Mullender $M$ et al: Early effects of mus cle atrophy on shoulder joint development in infants with unilateral birth brachial plexus injury. Dev Med Child Neurol, 2011; 53: 173-78

15. Calvo M, Zhu N, Grist J et al: Following nerve injury neuregulin-1 drives microglial proliferation and neuropathic pain via the MEK/ERK pathway. Glia, 2011; 59: 554-68

16. Li Y, Lein PJ, Ford GD et al: Neuregulin-1 inhibits neuroinflammatory responses in a rat model of organophosphate-nerve agent-induced delayed neuronal injury. J Neuroinflammation, 2015; 12: 64

17. Nicolino $S$, Panetto $A$, Raimondo $S$ et al: Denervation and reinnervation of adult skeletal muscle modulate mRNA expression of neuregulin-1 and ErbB receptors. Microsurgery, 2009; 29: 464-72

18. Sandri M: Autophagy in health and disease. 3. Involvement of autophagy in muscle atrophy. Am J Physiol Cell Physiol, 2010; 298: C1291-97

19. Soldado F, Fontecha CG, Marotta $M$ et al: The role of muscle imbalance in the pathogenesis of shoulder contracture after neonatal brachial plexus palsy: A study in a rat model. J Shoulder Elbow Surg, 2014; 23: 1003-9

20. Gallart-Palau X, Tarabal O, Casanovas A et al: Neuregulin-1 is concentrated in the postsynaptic subsurface cistern of C-bouton inputs to alpha-motoneurons and altered during motoneuron diseases. FASEB J, 2014; 28: 3618-32

21. Popadic Gacesa J, Dragnic NR, Prvulovic NM et al: The validity of estimating triceps brachii volume from single MRI cross-sectional area before and after resistance training. J Sports Sci, 2011; 29: 635-41

22. Narici M, Kayser B, Barattini P, Cerretelli P: Effects of 17-day spaceflight on electrically evoked torque and cross-sectional area of the human triceps surae. Eur J Appl Physiol, 2003; 90: 275-82

23. Coppey LJ, Gellett JS, Davidson EP et al: Effect of M40403 treatment of diabetic rats on endoneurial blood flow, motor nerve conduction velocity and vascular function of epineurial arterioles of the sciatic nerve. Br J Pharmacol, 2001; 134: 21-29

24. Gilliatt RW, Goodman HV, Willison RG: The recording of lateral popliteal nerve action potentials in man. J Neurol Neurosurg Psychiatry, 1961; 24: 305-18

25. Pondaag W, van der Veken LP, van Someren PJ et al: Intraoperative nerve action and compound motor action potential recordings in patients with obstetric brachial plexus lesions. J Neurosurg, 2008; 109: 946-54 